\begin{tabular}{|c|c|}
\hline Title & Toward a pluralistic conception of resilience \\
\hline Author(s) & Convertino, Matteo; V alverde, L. James, Jr. \\
\hline Citation & $\begin{array}{l}\text { Ecological Indicators, 107, } 105510 \\
\text { https://doi.org/10.1016/.ecolind.2019.105510 }\end{array}$ \\
\hline Issue Date & $2019-12$ \\
\hline Doc URL & http:/hdl.handle.net/2115/83729 \\
\hline Rights & $\begin{array}{l}\text { () 2019, Elsevier. This manuscript version is made available under the CC-BY-NC-ND } 4.0 \text { license } \\
\text { http://rreativecommons.org/icenses/by-nc-nd/4.0/ }\end{array}$ \\
\hline Rights(URL) & https://creativecommons.org/icenses/by-nc-nd/4.0/ \\
\hline Tyре & article (author version) \\
\hline File Information & Resilience Manuscript-Convertino- and-V alverde A pril2019notracks.pdf \\
\hline
\end{tabular}

Instructions for use 


\title{
Toward a Pluralistic Conception of Resilience
}

\author{
Matteo Convertino ${ }^{1,2}$, L. James Valverde, $\mathrm{Jr}^{3}$ \\ ${ }^{1}$ Nexus Group, Graduate School of Information Science and Technology, \\ Hokkaido University, Sapporo, JP \\ ${ }^{2}$ GI-CORE Global Station for Big Data and Cybersecurity, Hokkaido \\ University, Sapporo, JP \\ ${ }^{3}$ U.S. Department of Energy, Headquarters, Washington, DC \\ June 24, 2019 \\ $9 \quad$ Keywords: resilience, decisions, intentionality, foundations, systemic risk, networks, un- \\ ${ }_{12}^{*}$ *orresponding author contact: matteo@ist.hokudai.ac.jp
} 10 certainty

11 


\section{${ }_{13}$ Contents}

${ }_{14} 1$ Introduction 4

$\begin{array}{llll}15 & 2 & \text { Risk and Resilience in Complement } & 7\end{array}$

${ }_{16} \quad 2.1 \quad$ On the Uses (and Abuses) of Linearity . . . . . . . . . . . . . . . . . . 7

$17 \quad 2.2$ Models of Risk and Resilience . . . . . . . . . . . . . . . . . . . . . . . . . 10

$18 \quad 2.3$ System Dynamics and Resilience . . . . . . . . . . . . . . . . . . . . . 11

193 Steps Toward a Formal Conception of Engineered Resilience 13

$20 \quad 3.1$ Initial Steps, Towards a Plurality of Possible Destinations . . . . . . . . . . . 13

${ }_{21} \quad 3.2$ Time, Information, and Resilience $\ldots \ldots \ldots \ldots \ldots \ldots$

$22 \quad 3.3$ Criticality, Predictability, and Resilience . . . . . . . . . . . . . . . . . . . 19

$23 \quad 3.4$ System Landscape, Management, and Resilience . . . . . . . . . . . . . . . . 21

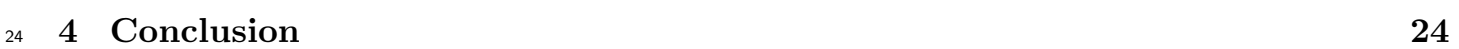




\begin{abstract}
The concept of resilience occupies an increasingly prominent position within contemporary efforts to confront many of modernity's most pressing challenges, including global environmental change, famine, infrastructure, poverty, and terrorism, to name but a few. Received views of resilience span a broad conceptual and theoretical terrain, with a diverse range of application domains and settings. In this paper, we identify several foundational tenets - dealing primarily with intent/intentionality and uncertainty - that are seen to underlie a number of recent accounts of resilience, and we explore the implications of these tenets for ongoing attempts to articulate the rudiments of an overarching resilience paradigm. Firstly, we explore the complemental nature of risk and resilience, looking, initially, at the role that linearity assumptions play in numerous resilience frameworks found in the literature. We then explore the limitations of these assumptions for efforts directed at modeling risk and resilience in complex domains. These discussions are then used to motivate a pluralistic conception of resilience, drawing inspiration and content from a broad range of sources and empirical domains, including information, network, and decision theories. Secondly, we sketch the rudiments of a framework for engineered resilience, the primary focus of which is the exploration of the fundamental challenges that system design and system performance pose for resilience managers. The conception of engineered resilience set forth here also considers how challenges concerning time and predictability should factor explicitly into the formal schemes that are used to represent and model resilience. Finally, we conclude with a summary of our findings, and we provide a brief sketch of possible future research directions.
\end{abstract}


Mahatma Gandhi

49

\section{Introduction}

Our modern preoccupation with resilience arises out of a basic human need to endure. In recent years, a host of scholars and practitioners — such as Levin (1999); Folke (2006); Levin and Lubchenco (2008); Carpenter et al. (2012); Linkov et al. (2014); Troell et al. (2014); Ganin et al. (2016); Goel et al. (2018); Linkov et al. (2018); Massaro et al. (2018); Rocha et al. (2018); Scheffer et al. (2018), Linkov and Trump (2019), and van Strien et al. (2019)

- have sought to outline the conceptual rudiments of an emerging "resilience paradigm". Constructive efforts such as these - directed, as they are, at integration, synthesis, and (in some instances) prescription - represent reasoned attempts to assimilate and make use of what has become an increasingly disparate array of conceptual schemes, methodologies, and worldviews. By their very nature, these "paradigm-building" efforts are replete with choices - choices (and, indeed, meta-choices) that shape the definition and scope of the emerging paradigm, and that influence, ultimately, its applicability and usefulness to human and ecological affairs. An exploration of the burgeoning literature that surrounds the topic of resilience reveals a congealing set of foundational tenets that conceptually ground many contemporary accounts of resilience. For our purposes here, we single out three tenets that are seen to underly an increasing number of received views of resilience:

T1 Utilitarian Orientation. Within the theoretical landscape of many contemporary accounts of resilience, the need or quest for resilience is typically construed as a desirable or sought-after end-in-itself. Such a mindset - decidedly utilitarian in its orientation — is in contrast to conceptualizations that look, for example, to contextualize the notion of resilience by situating it within larger theories or accounts of collective action, selforganization and emergence, and human intentionality.

T2 Monolithic Approaches to Reasoning About Uncertainty. Most formal accounts of resilience utilize the language of probability to reason about uncertainty, making use of a diverse range of probabilistic representations and methodologies. While understandable, given the numerous successes that probabilistic methods have enjoyed (across a diverse range of disciplines and problem domains) in recent decades, the monolithic status that probability theory enjoys within the resilience literature ultimately 
comes at the cost of a constrained vision of how uncertainty, in all its guises, might best be managed in a diverse range of resilience-related settings and contexts ${ }^{1}$

T3 Circumscribed Accounts of Human Cognition and Intentionality. Most contemporary accounts of resilience pay lip service to the idea that human beings often exhibit cognitive biases that impose limits or constraints on their ability to reason coherently under uncertainty, even in relatively simple choice situations (e.g., simple gambles, etc.). While the recognition of such biases is certainly relevant to the study of resilience, a myopic focus on the limitations of human cognition has the effect of rapidly shifting the focus away from cognition, broadly construed, towards, for instance, formal decision aides capable of minimizing the potentially deleterious effect of these biases on decision quality. In so doing, however, what often ends up being excluded from numerous contemporary accounts of resilience is the explicit consideration of matters pertaining to experimentation/observation, perception, and representation, together with nuanced treatments of human intentionality.

In what follows, we look to explore how tenets $\mathrm{T} 1, \mathrm{~T} 2$, and $\mathrm{T} 3$ are currently being construed and pursued within important strands of the resilience literature. Central to our objectives is the desire to offer a constructive critique of important aspects of how these construals and directions are currently shaping the research agendas and questions that underlie numerous ongoing scientific research programs that address the topic of resilience. In this regard, we shall argue that these tenets exert an influence that is - both individually and collectively - overly constrained in its purview, and that ultimately limits the usefulness of the resilience-related conceptual schemes and methodologies that emerge from these efforts.

From the outset, we note that many of the problems that we discuss here arise, in the first instance, from a failure to acknowledge that resilience is a concept whose theoretical bases lie not with just one "paradigm" or weltanschauung, but rather a plurality of conceptual schemes and viewpoints. This expansive viewpoint enables us to capture the complex interplay of natural/physical phenomena, as well as important aspects of human behavior and intervention, using a diverse panoply of descriptive, explanatory, and predictive tools. Figures 1 and 2 highlight the generality and methods of this viewpoint, seen through a set of conceptual lenses that are anchored in information, network, and decision theories. Figure 1

\footnotetext{
${ }^{1}$ It is worth noting that numerous contemporary accounts of resilience often hover near this conceptual terrain when they probe the nature of uncertainty itself — with a number of commentators arguing, for example, that there exist fundamental limitations in our ability to make predictively informative assertions about large-scale socio-biological and technical systems. These limitations are often taken to have important implications for any well-motivated theory or conception of resilience; in this regard, later in our discussion, we offer some perspectives on the topic of predictability and its relevance to our evolving conceptions of resilience.
} 
shows how human decisions give rise to emergent scale-free networks; Figure 2 depicts the variables and probabilistic patterns that allow us to formally characterize complex systems, dependent on desired performance and systems' drivers. By pursuing this path, we look to broaden the field of vision that is brought to resilience-related challenges and concerns, in ways that ultimately enable a pluralistic conception of resilience to emerge.

The discussion that follows is divided into three parts. In the first part, we explore the complemental nature of risk and resilience. We begin this discussion by considering, first, the role that linearity plays in many prevailing accounts of risk and resilience. This discussion is then used to motivate a more general discussion concerning the challenges entailed in modeling risk and resilience, in a broad range of empirical settings and contexts. We close this section with an outline of the conceptual rudiments of a pluralistic approach to reasoning about resilience. In the second part, we sketch the rudiments of a theory or conception of engineered resilience. We begin this portion of our discussion by confronting the conceptual and practical limitations of tenets T1 and T3. Specifically, we explore aspects of an idea that is seen to underlie many contemporary debates surrounding the notion of resilience, namely, that system design - and, by implication, optimal system performance (Figures 2 and 3 show probabilistic and time-dependent patterns of systems' performance) — is achieved via resilience only. As part of this discussion, we go some distance towards countering this view by exploring resilience frameworks and application domains where optimal system design is seen to require (i) an awareness and understanding of complex stakeholder preferences and value trade-offs; (ii) a multifaceted understanding of outcomes and consequences; and (iii) a holistic understanding of what it means to optimize overall system performance. As part of this discussion, we explore how time factors into our broadened conception of resilience, and we take up matters pertaining to to criticality and predictability in our characterization and evaluation of complex systems. Throughout our discussion, we endeavor to cast a wide field of vision — both conceptually and methodologically — and we provide illustrations drawn from a diverse range of doamins and empirical settings. In addition, we explore recent theoretical and computational advancements in the study of resilience for socio-biological and technological systems, from the perspective of complex systems science (see, e.g., Bialek et al. (2001), Prokopenko et al. (2008), Marsili et al. (2013), Helbing et al. (2015), and BarYam (2016)). Furthermore, we seek to broaden the typically encountered thematic focus on infrastructure, by also including alternative views drawn from studies of environmentallydependent, multiscale socio-biological systems. Finally, we conclude with a summary of our findings and a brief discussion of possible future research directions. 


\section{Risk and Resilience in Complement}

The Latin word resilio means to "rebound" or to "spring back" — and, indeed, our ordinarylanguage usage of the word "resilience" is consistent with this etymology. In contrast, risk is typically defined as the likelihood that a stressor affects a given system, considering that „system's vulnerabilities, as well as its dynamics in space and time (Kéfi et al., 2013; Kefi et al. 2014). Accordingly, resilience can be viewed as the observed or predicted response of a system to one or more definable risks. In this way, risk and resilience are easily seen as complemental notions, with a conceptual interplay that is, at once, both common-sensical and capable of yielding important insights about complex systems, especially at systemic levels of aggregation (Helbing et al., 2015) 2 In this section, we explore aspects of the complemental nature of risk and resilience, beginning with an exploration of how our assumptions concerning linearity play into our descriptions and representations of resilience. These considerations then lead us to a more general discussion of the challenges associated with modeling risk and resilience. We close this section with a tentative outline of the rudiments of a pluralistic conception of resilience.

\subsection{On the Uses (and Abuses) of Linearity}

Linearity is to science as, perhaps, concrete is to civil engineering and construction. Often invoked as a convenient fiction, linearity assumptions are typically used to render systems that are otherwise in-amenable to decomposition and analysis (due to, say, inherent system complexities and/or attendant uncertainties), amenable to first-order approximation and evaluation. While oftentimes a sensible starting point in the analysis of complex systems, the invocation of linearity assumptions can sometimes obfuscate and oversimplify, to the point where erroneous (in some instances, even potentially dangerous) prescriptions can emerge, requiring careful interpretation and bracketing. For our purpose here, we adopt the most general definition of complex systems: systems whose cause-effect dynamics is highly nonlinear and non deterministic. Such systems are, of course, less trivial and predictable than simple systems. Figure 4 illustrates the differences between linear and a non-linear systems, where components' interactions is a minor and predominant factor in systems' response, respectively. In the former and latter cases, a risk and resilience approach is suitable. Figure

\footnotetext{
${ }^{2}$ We construe risk and resilience in a manner that looks to eschew any kind of value- or norm-based hierarchy. In contrast, claims by researchers such as Linkov et al. (2014) that "resilience management goes beyond risk management" seem misplaced, in that they can be taken to imply a presumed hierarchy, with resilience somehow occupying a higher level of "importance", enjoying a primacy that appears grounded in the belief that in designing and managing complex systems, the desire or quest for resilience is somehow "most essential" or "more fundamental" than other goals, objectives, and desired end-states. In truth, there are no a priori reasons to suppose that such views are supportable on theoretical grounds; their prescriptive relevance derives purely from a value-laden understanding of human meaning and purpose in specific contexts and situations.
} 
5 shows how complex systems can be categorized into dynamical classes, based on how systems' components interact with each other and perform independently for achieving systems' functions. The quantification of the functioning of complex systems is always dependent on available data; therefore, any assessment should always consider the dependence of function ¡n the amount of information used that can reconstruct systems' networks (see, e.g., Servadio and Convertino (2018) and Li and Convertino (2019)).

A common difficulty with many of the resilience frameworks that researchers have sketched in recent years is the inherent linearity, in time and in space, of the examples that are often cited in this work. In many instances, resilience is interpreted or seen as a single risk-response function. For example, Linkov et al. (2014), and more recently Linkov et al. (2018), present case studies that are grounded in decidedly linear characterizations of potential system states. This simplistic view, while perhaps a useful starting point in such discussions, is in contrast to more frequently encountered (certainly in the types of real-world systems they cite as examples) non-linear system dynamics, where multiple drivers and events are considered over extended time horizons. Only in the simplest cases can resilience be assessed or measured by looking at just one instantaneous factor or event and its effects. An example of spatial nonlinearity is provided in Figure 7], where the community interdependence network (inferred by the model developed in Servadio and Convertino (2018)) is applied to epidemiological time series of Leptospirosis in Sri Lanka (Convertino et al. 2019). This example shows how space and time are, indeed, connected and non-linear scale-free time series, representative of epidemic critical states (depicted in the top plot), correspond to scale-free transmission networks; vice versa endemic states are related to seasonal time series and exponential random networks. This example typifies a line of reasoning that highlights the fact that resilience cannot be assumed as a linear function as assumed by analytic frameworks and models that claim to deploy the "science of resilience" in practical applications (see Linkov and Trump (2019)). Moreover, resilience should not be evaluated solely in terms of "speed of recovery" to some previous system state, before the influence of any stressor(s); instead, resilience should also be evaluated in terms of the magnitude of effects, together with the full range of possible state transitions via probability distribution functions, including transitions toward "better" or perhaps preferred system states (see Figure 3). This probabilistic mapping of systems' dynamics allow us to create the system potential landscape (Figure 8) that describes and represents all likely systems' states, dependent on data-inferred dynamics and stakeholders' mental models (including model choice(s) and preferences).

Experience teaches us that low risks can actually give rise to major impacts on systems 


\footnotetext{
${ }^{3}$ An important early example of this line of reasoning is found in Charles Perrow's seminal book, Normal Accidents.

${ }^{4}$ In mechanical systems, for example, an engineered product is evaluated for resilience by testing it under the same cyclical conditions, observing the systems' responses over the time horizon for which the product's functions need to be guaranteed. Such tests have obvious analogues within the realms of complex socioecological systems

${ }^{5}$ Systemic risk (Beale et al. 2011 Haldane and May, 2011 Helbing 2013) can be defined as the likelihood of an outcome (typically adverse/undesired), evaluated by taking into account local vulnerability and systems' interdependencies in space and time. Assessment of systemic risk typically entails considering multiple risks that are capable of affecting the magnitude of aggregate outcomes (such as multiple diseases, group behavioral dynamics, flooding, etc.) for a specified time horizon. More generally, performance can be evaluated considering systemic risk and attendant costs.
} 
systemic risk landscape, as well as the ability for agents (e.g., affected populations) to learn and adapt (where and when possible). In this way, resilience is, perhaps, more akin to a "trajectory" (Figure 3) — thereby better represented by slopes of response and recovery, depth of the system response curve, and post-shock values for system function over time horizons deemed important or relevant for intervention and control. In truth, response curves and system potential landscapes are partial elements of what system-level resilience is. We now consider how the evolution of system performance should, in probabilistic terms, be the risk-independent pattern to consider when evaluating systemic resilience.

\subsection{Models of Risk and Resilience}

The nature of the relationships that can be said to exist between "models" and "reality" is, of course, a topic that has preoccupied philosophers and scientists, alike, for centuries. A review of the salient themes that emerge from this body of thought is well beyond our scope here suffice it to say that we accept that the nature of the relationship between any model and the "reality" it seeks to describe or represent is necessarily tenuous. To the extent that this characterization is accurate, it is surprising to note that numerous contemporary accounts of resilience seem to somehow lose sight of this point. This idea is most prevalent within certain ideological camps (e.g., computational scientists), and it typically finds expression in a line of thought that supposes that if a model is capable of generating highly accurate predictions, then the embedded processes represent (or at least reflect) the "true" predicted processes. Munoz-Carpena et al. 2013, for example, promulgate the view that "more information is better". This principle has its origins in the classical reductionist belief that the successive accumulation of knowledge leads to closer and closer approximations of reality. A vast array of empirical insights, derived from a range of scientific disciplines, teach us, however, that more information can lead to more uncertainty. Accordingly, managing information value (Feistel and Ebeling, 2016) is an important prerequisite to effective problem solving and decisionmaking within the realm of complex systems. Furthermore, consideration of trade-offs that exist between sensitivity, uncertainty, and complexity of information is a common problem within existing decision-making paradigms, where perfect information is seldom available to decision-makers.

A wide range of stochastic decision-making models have been proposed in the literature that focus on modeling complex systems under uncertainty (Shalizi and Crutchfield, 2001. Marsili et al., 2013, Helbing et al., 2015). This strand within the literature of work teaches us many things - for example, that any model is an information machine (Marsili et al. 2013: Quax et al. 2016), with its own variability, uncertainty, and complexity. In order to 
analyze risk and resilience, the key is to have models that are capable of optimizing the important trade-offs that exist between these features (which often exhibit non-linearity). In such contexts, non-linearities can arise any number of ways. For example, the magnitude of a system's performance (gathered from data, or as an output of models) may be uninformative about the magnitude of a given hazard and its risk in such instances; it is important to also consider the significance of small changes in input factors that can potentially have dramatic influences on performance. For instance, small, gradual changes in input factors that accumulate over time and space can bring about cascading changes in interconnected system performance metrics (e.g., numerous population outcomes that are related to one single cause). This "butterfly effect" (as it is often described in the chaos theory literature see, e.g., Crutchfield (2012)) is the potential for a ripple in one part of a system's "world" to be amplified and subsequently lead to major disturbances in another part of the system (due to the increased connectivity of system parts and multiple, interconnected systems) is another symptom of non-linearity. This type of phenomenon is, of course, commonplace within many biological systems, where, for instance, numerous biomarkers are highly interconnected and even small changes may be extremely meaningful for overall system performance in the longterm (see, e.g., Convertino et al. (2018)). At a much larger scale, consider the case of many interdependent infectious diseases that are related to the same environmental and social causes, leading to co-occurrent disease transmission (see, e.g., Convertino et al. (2014)).

\subsection{System Dynamics and Resilience}

Proper characterization and evaluation of system response is central to any well-motivated approach to resilience. Adding, then, to our observations above concerning non-linearities, it is important to recognize that risk is not solely proportional to the depth of the system response curve, as this reflects a system's outcome as a function of ex post interdependent hazards whose intensity may or may not be well predicted ex ante ${ }^{6}$ This observation is important, because unexpected events - and possibly other unknown factors - can hardly be anticipated with perfect foresight, despite our best efforts to eliminate risks and to include all salient factors. Instances where risk is seen to be proportional to the depth of the system response curve typically arise when the system response curve is constructed using historical data, focusing on correlations with one single hazard. The level that is reached by the system after recovery, and the system's ability to withstand or rebound faster after an identical shock at future times, is but one of several crucial elements that should be evaluated when assessing resilience and system criticality. More generally, we must seek to characterize and evaluate

\footnotetext{
${ }^{6}$ This point is often overlooked in the literature - see, e.g., Linkov et al. (2014).
} 
- Systemicity. Consistent with our earlier discussion, the resilience of a complex system is not just the response of that system to one well-identified hazard, but rather, the response of that system to multiple connected hazards, plus any intrinsic ability of that system to increase fitness. In situations where one hazard is identified, resilience is a non-linear function of risk, where risk is not solely proportional to the magnitude of the attendant hazards, but also considers its probabilities and vulnerability functions (including exposure factors), convoluted to some uncontrollable noise. Equivalent stressors can potentially give rise to a very different response; only a normalization of system functionalities can make systems comparable in terms of resilience $]^{7}$

- Spatio-Temporal Non-Linearity. In looking to formally characterize resilience, history often matters, i.e., resilience is typically history-dependent. More specifically, resilience is dependent non-linearly on the present, the past, and future sensed risks. Indeed, oftentimes, the larger the realized risk, the larger the resilience of the system "the more we fall the more we learn". System performance achieved after a disturbance (e.g., the slope of, and area under, the system's functionality curve, and post-stress performance) can change non-linearly; thus, the very same combination of risk factors can lead to different resilience levels, and vice-versa. Small risks typically accumulate critically and generate systemic effects after cascading events on spatio-temporal connections of complex systems. Notwithstanding this non-linearity, the higher the controlled ability of a system to change to multiple varying states (desirably around optimal states), dependent on environmental fluctuations, the higher the resilience.

- Subjectivity. Resilience is not solely proportional to one functional or structural cri-

\footnotetext{
${ }^{7}$ Consider, for instance, the case of a hurricane of the same intensity level hitting two nearby but very different locations; or a psychological stress affecting two individuals linked by family ties, but nurtured in very different environments. A wealth of social science theory and empirical case study teaches us that socio-environmental context exerts a tremendous influence in the response of communities and individuals to the same stressors. The detailed characterization of heterogeneities is therefore fundamental for predicting complex systems, and for comparing them after their normalization, dependent on the key heterogeneities leading to different outcomes.
} 
teria (e.g., complementary damage, speed of recovery, etc.), but also to a stakeholderweighted multi-criteria function that captures desired system performance, stakeholder preferences on performance drivers, and the quality of information related to performance and disturbances. Quality of information, as much as other "intangible" criteria, constitute the "subjective" components of resilience, beyond its strictly "objective" features. In this way, so-called "cup and ball" diagrams that are commonly found in the literature only reflect the structural components of resilience - and thereby omit critical features of many real-world systems.

\section{Steps Toward a Formal Conception of Engineered Re- silience}

\subsection{Initial Steps, Towards a Plurality of Possible Destinations}

Coming out of the discussion above, what we now seek is the beginnings of a conceptual outline for an enriched vision of resilience, where human intentionality is seen to play a central, defining role. In what follows, we explore aspects of what it means to, in effect, engineer resilience. In so doing, we draw inspiration and insights from a range of disciplines, including complexity science, information theory, network and decision-theoretic sciences, together with an appreciation for what it means to apply these concepts in a diverse range of settings and contexts. Ultimately, we seek a conception of, and approach to, resilience that is capable of serving a host of purposes, including:

- Helping life (at any scale of biological organization) to flourish sustainably on Earth;

- Protecting and providing thoughtful/purposeful stewardship of the Earth's atmosphere and ecosystems;

- The health and protection of people and property; and,

- The ability to sustain infrastructure that is essential to the proper functioning of our increasingly technological society and it socio-economic systems.

All of these interconnected purposes, which reflect a "safe operating space for humanity" (Rockström et al. 2009), are (and indeed must be) centered on a pluralistic conception of resilience that encompasses both self-organization and intentionality of social actors and complex systems. Pursuing such expansive ends requires that, independent of the size of the complex system considered, the interconnections of a given system with all others must 
be taken into account (to the extent that knowledge and resources allow). Increasingly, such concerns are intertwined with more general societal desires or quests for sustainability; we argue that any model of sustainability must consider forms of resilience that aim for consistently improving desired ecosystem services versus risk-based approaches that focus on maintaining current levels of desired services.

At its essence, a model is a representation of how a system is "seen" and described. The sensitivity of a system's features is defined by the so-called ST-scale that determines the spatial and temporal lens of analysis. In a broader sense, cognition, ST-scale, and entropy are the "how, where/when, and how much" that a system is analyzed. At the bottom of Figure 2 the left plot is a single risk-dependent performance profile (deterministic), the middle plot is a probabilistic performance profile, dependent on one single system's driver, and the plot on the right is a risk-independent probability distribution of performance.

The conceptual relevance and practical utility of our resilience framework is borne out in a diverse range of empirical settings and contexts. It is instructive to consider examples drawn from both natural and engineered systems. In the context of socio-environmental systems, considering data and numerical simulations of Hurricane Katrina (2005) and Hurricane Sandy (2012), the (ex post) resilience of New Orleans and NYC can be evaluated by considering (i) the urban and natural ecosystem's ability to respond early; (ii) the damage in terms of structure and function; and (iii) the speed of recovery (Bonanno et al., 2007; Shultz et al., 2007; Pietrzak et al., 2014; Valverde and Convertino, 2019). Interestingly, the same basic concepts and approach finds application, for instance, in the study of infectious diseases, with high and low frequencies of occurrence, in cases of foodborne and Ebola outbreaks, respectively.

In the case of repetitive events, it is reasonable to expect that populations are capable of learning, over time, how to be more resilient to equivalent (or at least "similar) events. In this vein, an interesting example is the state of Florida, which, in light of its recurring tropical cyclone season, has put in place an efficient surveillance system for rapid response and recovery. Along similar lines, other examples include flood control infrastructure and runoff monitoring, which work effectively to reduce extreme runoff events. Within the realm of public health, examples include the surveillance, hygiene, and sanitation infrastructure put in place in developed and developing countries that are affected by waterborne diseases, such as cholera (e.g., Bangladesh and Haiti are instructive examples of populations that have built effective response mechanisms). Also worthy of note, in terms of system function, are the networks of epidemiological surveillance of infectious diseases worldwide - e.g., FOODNORS for foodborne outbreaks in the USA, and ProMED-HealthMap for infectious diseases 
at the global scale. As part of their design, these systems seek continual improvement, and have demonstrated an ability to minimize the incidence of massive outbreaks. All these examples show how realized risks were necessary elements to building resilience over time; moreover, they show that a purely ideological "risk-free" resilience approach does not exist.

Of course, learning systems such as those referenced above need to be maintained and updated on a regular basis, taking into consideration natural and anthropogenic variability (e.g., climate extremes, agricultural intensification, urbanization, and related factors). These examples illustrate that active surveillance of system structure and function (e.g., supply chain integrity/reliability and foodborne infection cases) is crucial to building and maintaining resilience, and to avoiding catastrophic events. Ultimately, it is history that teaches us that both positive and negative events are necessary to build resilient systems ${ }^{8}$

Within the context of a more traditional risk-based framework, the change from one microstate to another is typically associated with an alteration of system function, observable in the increased variability of system components (color of node from white to red), and for major transitions also in the variability of system structure (e.g., connectivity among nodes) (Figure 3). Before any tipping point, the variance of system function is increasing while the stable state corresponds to a low variance state (e.g., network with "white nodes").

From an information-theoretic perspective, similar transitions have been observed in social systems (Borge-Holthoefer et al., 2016), where approaching critical states (a manifestation of critical dynamics) implies an increase of fluctuations in the information exchange at the system scale, after accumulation of local fluctuations above a critical threshold. These fluctuations are typically responding to time-point hazards and do not necessarily reflect the system's long-term performance. An intuitive example of this is the hyperactivity of certain physiological biomarkers that an individual presents during intense exercise; fluctuations of all sizes occur until a peak performance level is reached, and after they slow down to baseline condition levels. These dynamics and fluctuations do not reveal anything about the long-term — for instance, the lifetime performance of the individual considered. Traditional risk analysis has been mostly focused on these time-point single hazard-dependent events, rather than having a long-term view that is more in line with resilience paradigms that are focused on guaranteeing a positive resilient trajectory, with increasing systems' performance, independent of any preconceived risk.

Exploring these matters more broadly, we note that resilience is an innate property of any living system, arising from the evolutionary pressures of survival, and giving rise, over the

\footnotetext{
${ }^{8}$ Interestingly, this is an idea that is reflected, in a negative way, by the current "exposomics" and epigenetics theories of disease generation in populations, where a multiplicity of factors contribute to the health of an individual (J Patel and K Manrai, 2015).
} 
long term, to an optimal pruning of coupled form and function (Bak, 2013; Banavar et al. 2014). In this context, resilience is seen to depend on both the interconnected structure and function of systems, where structure is determined by the physical interconnections of system components, and function is the stakeholder-independent endogenous byproduct of complex systems. For biological systems, a classical example is the brain, where structure is determined by the dendritic assemblage of different neurons at the micro-scale, and where function arises from the electrical activity among many neurons for supporting other system's functions (e.g., brain dynamics at the meso- and macro-scale) (Damasio and Carvalho, 2013). In this way, the brain is the central "system of systems" for human beings, responsible for controlling all physical and functional processes.

In natural systems, the same duality of form and function is observed (West et al., 1997 Banavar et al., 1999; Bak, 2013, Banavar et al. 2014, Tendler et al., 2015, Seoane and Solé, 2015: Koçillari et al. 2018). For instance, in river systems, structure is defined by the river network, with optimal and ubiquitous features, whereas function is the water transport mechanisms from a hydrological viewpoint, and so on and so forth, for geochemical and ecological processes that become apparent by enlarging the purview of analysis. All of these systems have embedded within them a natural capacity for building resilience over time, considering both the capacity to withstand structure-forming shocks and the long-term drive toward optimality (Hidalgo et al. 2016). Interestingly, for any individual, the human brain is part of the "collective", or part of the "aggregate societal brain", at the population scale, which is also determining (or at least influencing) the trajectories of natural and man-made systems. In this sense, it is important to broaden the traditional field of vision that is brought to such problems, to include consideration of the anthropogenic dynamics of resilience for any system at the population scale (Diamond, 2005).

Our discussion thus far suggests that by enlarging the lens of analysis, it is possible to observe coupled structures and functions simultaneously, but more importantly, to identify the relevant information at any scale of analysis. In the case of river systems, for example, a network of dams and locks is seen to provide services to human populations, including flood control, hydroelectric energy, and transportation. As Linkov et al. (2014) and others have suggested, all of these systems should be considered in toto, with complexity and network theory providing useful analytic vehicles for exploring the structural and behavioral modalities of such systems. For its part, complexity theory has much to teach us about resilience. By simplifying the analysis of systems just enough to make possible the discernment of important system drivers at different scales, complexity theory yields insights that are useful for design and management purposes. From a decision-making perspective, it is useful to 
recognize those situations where only a subset of drivers are important at the system-scale, and where single component drivers are of second- or lower-order importance in relation to the scale and objectives considered. This extraction of information can be accomplished using global sensitivity and uncertainty analysis models that identify the important information for the stated objectives. It is often the case that the emergence of systemic patterns arises only from a subset of critical drivers, whose importance and interaction with other factors is crucial to understanding the behavioral modalities and dynamics of the system. This holistic understanding of complex systems can be achieved by exploring the whole system landscape of potential states and their drivers, with emphasis on the dynamical trajectories and stressors that lead to emergent patterns (Figures 3 and 7 , the patterns depicted in the latter figure illustrate the typical probability distribution function of infectious disease cases, in the form of power-law and exponential distributions, corresponding to epidemics and endemics, respectively).

\subsection{Time, Information, and Resilience}

At a foundational level, it is reasonable to suppose that time should factor prominently in the theories and conceptual schemes that we devise to address resilience-related challenges and concerns; in truth, however, this topic has been given scant attention in the literature. Philosophers have, of course, long preoccupied themselves with the nature of the relationship that human beings have with time. Though such discussions are somewhat removed from our concerns here, an awareness and understanding of how humans perceive, experience, and value time can meaningfully guide our efforts to broaden the conceptual terrain that contextualizes and informs our understanding of resilience.

We begin this portion of our discussion by considering the situation where one or more individuals (say, e.g., the "resilience managers" that Linkov et al. (2014) envision) are tasked with creating and/or sustaining system-level resilience; such individuals must, as a matter of necessity, come to understand that resilience must be viewed through the a complementary set of lenses that partition time into various time horizons — ranging from the immediate to the long-term. As an example, take society's desire for population resilience, and the events surrounding Hurricane Katrina in 2005 as a specific case in point. Some have argued Cutter et al. 2013, Tierney, 2014) - rightly so, we believe - that Hurricane Katrina was, in fact, a necessary event for building resilience over time in that geographic region. The probative portions of this argument require a general systems theory perspective, together with a holistic view of collective action, taking into account all relevant factors affecting the built vs. natural environment, together with an understanding of the attendant influence that 
these factors and events have on political institutions and stakeholders' ability to effect change directed at the development of effective flood protection systems. Similar arguments can be made about Hurricane Sandy and the 9/11 terror attacks (Sarapas et al., 2011, Valverde and Convertino, 2019). To be sure, arguments of this nature are inherently difficult, given that we typically know more about the past than about the future — which in turn complicates efforts to interweave causation and intervention in ways that give rise to desirable end-states over time. This said, it is perhaps an inevitable feature of human existence and mankind's (seemingly insatiable) desire for material progress that the discovery and elimination (or minimization) of points of failure cannot occur without the occasional occurrence of negative — sometimes even catastrophic — events. In this way, failure constitutes an inalienable element of resilience, understood vis-a-vis the arrow of time; given this line of thought, it is not surprising that much of the resilience management literature is grounded in theories of adaptive management (Holling and Gunderson, 2002, Convertino et al., 2013), which mimics the adaption, for biological systems, to fast and slow external changes.

Given these considerations, it is interesting to observe that many contemporary accounts of resilience seem predicated on the idea that resilience is only an inherent, though perhaps ultimately manageable, property of systems. Indeed, the notion of resilience as an "emergent" property of systems (Kauffman, 1993, Jiménez et al. 2008; Anderson et al., 2013, Seoane and Solé, 2015, Tendler et al., 2015; Lansing et al. 2017) is strangely absent from several recent characterizations of the concept (see, e.g., Linkov and Trump (2019). As we have already noted in portions of our discussion above, biological science has much to teach us about this kind of phenomena. Traversing the micro-to-macro cellular life and population scales reveals instances where resilience is a continually evolving process, involving consideration of the intertwined evolution of human and natural systems. Human history is, of course, replete with examples of populations who have learnt, over time, how best to respond to flooding, fires, crime, outbreaks of infectious and chronic diseases, war, and other largescale events (Diamond, 2005). There are aspects of this learning process that are overt and intentional, and others that are less intentional, arising, sometimes, by accident or through trail-and-error. Given the ever-expanding reach of data collection and analysis, combined with burgeoning advances in artificial intelligence, computational science, sensor technologies, and global system science, it is reasonable to suppose that the window of unpredictability about past risks (i.e., "known knowns") will be narrowed (though, of course, perhaps never entirely closed), but new risks will emerge in relation to innovation and surprise. Almost surely, the unexpected/unanticipated will still occur, and perhaps with even greater frequency and/or severity (considering the dramatic changes the world is undergoing, e.g., climate 
change, technological innovation, globalization, etc.). Such occurrences are likely to defy expectations that are predicated on inferential mechanisms that presuppose stable historical baselines, structural regularities, and the like. The tightly coupled nature of these systems, together with the inherently "reflexive" nature of modern technological society (Beck, 2009), almost ensure that mankind will underestimate risks. Even more broadly, hypothetically, even our current resilient zeitgeist may be challenged, due to truly unexpected events that force us to revise our current information and values, with dramatically different information in turn leading us to new conceptual (and computational) frames and modes of discourse and analysis. The fundamental question that emerges from such possibilities is this: is there some critical information that is always valid and upon which we can always build upon? This is a line of questioning to which we now turn.

\subsection{Criticality, Predictability, and Resilience}

As discussed earlier, it is well understood that complexity theory provides a number of useful analogies to physical systems that can significantly aid efforts to (i) improve theoretical and computational models of these systems; (ii) understand system-level dependencies; and (iii) guide the design and monitoring of complex systems by considering inner criticalities and externalities. As instrumental as these models and frameworks are, there is a method-centric tone that runs through significant portions of the resilience literature, much of it motivated by a desire, on the one hand, to question the usefulness of traditional risk assessment tools for assessing/measuring resilience, and a desire, on the other, to call for new "frameworks and models enabling system-wide and network-wide resilience analysis" (Linkov et al., 2014). Taken as a whole, these strands within the literature are not altogether successful in putting across a coherent picture of resilience, for reasons which we now consider.

We begin by noting that the notion of "unpredictability" factors prominently in the narratives of both academic and non-academic commentators about resilience. Linkov et al. (2014), for example, argue that "traditional risk assessment tools are limited in their usefulness for quantitative analyses of resilience"; at the same time, these same authors offer prescriptions and illustrative case studies that seem distinctly rooted in traditional risk assessment frameworks and models.

Our prevailing conceptions of risk require careful consideration to both sides of the traditional risk equation — an awareness/understanding of threats and hazards must in some way be conjoined with ways to think and talk about consequential outcomes that play an important role in the kinds of societies that we wish to inhabit.

It is not unreasonable to suppose that the gradual abandonment of the Gaussian view of 
natural systems phenomena, combined with recently developed (and future) analytical and computational tools capable of providing the mapping of systems' probabilistic landscape (Figures 3 and 4) (i.e., systems' states and all trajectories considering all potential drivers and their combinations) will, over time, increase the predictability of events that are currently thought to be unpredictable.

Ultimately, we are inclined to believe that there are good reasons to suppose that "unpredictability" should not factor prominently in our willingness to accept the claim that traditional risk analysis tools "are no longer sufficient to address the evolving nature of risks in the modern world" (Linkov et al., 2014). In truth, we are inclined to believe that the dilemma that unpredictability poses in any discussion of resilience is not either/or, nor should it be seen to be conceptually or practically "fatal". The sensibility of this viewpoint can be argued a number of ways, including (i) the ability to render complex systems amenable to parsimonious analysis by exploiting recurrent patterns arising from universal topological features; (ii) the rareness of completely chaotic behavior versus critical dynamics $9^{9}$ and (iii) the oftentimes poorly-motivated quest for precision in analyzing extreme events. The latter point is, unfortunately, a bias of modelers who look to maximize model predictive accuracy in ways that are spurious (due, mainly, to the limitations of the data at hand) and that often fail to heed the precision-oriented limitations that system errors impose within complex systems. With sound models (conceptual, analytical, computational, and practical models), it is possible to scale-up small events to large events, along their power-law distributions, and to thereby estimate the frequency and intensity of potentially catastrophic events. An example where this approach has been applied successfully comes from the fields of hydrogeomorphology and hydroepidemiology, to predict large runoff and cholera events Bertuzzo et al. 2011; You et al., 2013, Convertino et al., 2014, Convertino and Liu, 2016). Zipf's law - a special power law with an exponent close to unity — is ubiquitously observed in nature. The inverse relation between rank and frequency of events implies the existence of a few frequent extreme patterns and numerous rare patterns. The origin and function of Zipf's law has been explored with respect to information processing in language and communication evolution, as well as numerous applications within natural systems. Zipf's law has also been observed in the activity patterns of real neural networks, though probing its functionality in the animal brain is a formidable task; these empirical findings suggest an incredible connection between optimal decision making and criticality that can be implementable in current intelligent computer systems. In this regard, some authors have identified a critical layer where the cluster size distribution of processed data obeys a reciprocal relationship between rank

\footnotetext{
${ }^{9}$ N.B. that even chaotic behavior displays stable attractors.
} 
and frequency. Deep learning ensures balanced data grouping by extracting similarities and differences between data. Furthermore, it verifies that data structure in the critical layer is most informative to reliably generate patterns of training data. Therefore, the criticality can explain the operational excellence of deep learning and provide a useful conceptual vehicle for probing optimal network architectures.

Where such considerations lead us, then, is that while we may never reach complete predictability for all of the events that currently (or may possibly) interest us, advancement of global sensitivity and uncertainty analyses, supported by information theory, can leverage "unpredictability" as a positive impetus for exploring all system states, and to find optimal design alternatives. The same predictive models can also be used for surveillance purposes (Convertino and Hedberg, 2014, Vilas et al., 2017) in order to rapidly detect early warning signals for potentially catastrophic events that clearly determine critical transitions (Scheffer et al. 2001, 2012).

\subsection{System Landscape, Management, and Resilience}

Our commentary above goes some distance towards suggesting that risk and resilience can, in certain respects, be seen as two sides of the same coin. For the design and management of complex engineering systems, risk factors can be identified as causal factors affecting resilience via analyses of data and the assessment of systemic-level risk(s) (Sheffi et al., 2005, De Weck et al., 2011; Helbing, 2013). Our ability to arrive at credible (and requisite) representations of systemic landscapes is a vitally important prerequisite to any reasoned attempt to develop sensible prescriptive theories and frameworks directed at the design and management of complex engineering systems. It is this final topic to which we now turn.

We begin by noting that portfolio approaches suggested in the literature incorporate systemic purviews of risk, and are capable of considering a multitude of scenarios for complex systems (see, e.g., Valverde and Convertino (2019)). Such frameworks can aid decision-maker efforts to identify the optimal design paths that support optimal form and function of systems in terms of resilience. Portfolio-based approaches to systems management typically embody dynamical models that reflect both the "blind watchmaker" dynamics of nature (criticality à la Bak) and causal external triggers, geared towards identifying optimal solutions that take into account the randomness and variability of system events. Portfolio approaches also consider the structure of the system analyzed, and they are capable of learning from previous events and outcomes; further, they enable decision-makers to make optimal decisions, based on both system structure and function, by identifying optimal design and management alternatives, after evaluating the (often) combinatoric space of potential alternatives, constrained 
by available budgets and resources. The scenarios considered are also unobserved scenarios — in contrast to reductionist models that only reproduce observations - that are used to explore a vast plurality of potential system outcomes for taking optimal decisions in the face of what is considered possible.

An in-depth understanding of potential system states can be built by taking advantage of uncertainty propagation methods on probabilistic computational models. For instance, global sensitivity and uncertainty analyses (GSUA) are perturbation methods that propagate uncertainty from system drivers to outcomes (e.g. performance); these are input and output factors in a model designed to predict system dynamics. An idealized example is provided in Figure 8, where two drivers - labelled F1 and F2 - can affect the whole system or just one of its components (it is possible to consider the whole system, or just a portion of it in the physical domain). More generally, it is important to recognize that global scale factors and outcomes are much like systemic scale factors; however, global factors are not necessarily created by systemic components (such as networks that connect communities), whereas systemic outcomes are systemically distributed. In this way, systemic outcomes can be global, but global outcomes are not necessarily systemic because they can be driven by concomitant local factors.

In the context of contagion phenomena (such as infectious diseases, but other examples include cyber-attacks, false information diffusion, and species invasion), outcomes are outbreaks over a region (a system's component can be a geographical area, while the whole system is the whole globe). F1 and F2 can be two different pathogenic drivers, such as Zika and Dengue viruses (hazards). Systemic factors could include, e.g., transportation networks, while local factors might include local weather factors that determine the environmental niche of pathogens (these can be vulnerability features, such as rainfall and landscape wetness index). Global factors can, for instance, be similar vulnerability features that are homogenous across all communities (e.g., lack of medical facilities). These common global factors can give rise to equivalent epidemics without the need of systemic factors, such as long-range human mobility networks. A good distinction between epidemics and pandemics is likely related to the presence of systemic networks which are the predominant contagion spreading feature of the latter.

Systemic performance is not necessarily associated to one single risk, but rather to multiple risks; therefore, resilience is not the complementary function of one single risk function. In Figure 8, state $A$ is characterized by the dominance of one stressor that acts locally (e.g., due to local weather) and produces local outcomes (such as a local endemics). These local outcomes are typically associated by a bimodal distribution that, for instance, corresponds 
to seasonal dynamics. State B is characterized by the coexistence of two stressors at the local and global scale. State $\mathrm{C}$ is characterized by the predominance of one single stressor that is systemic. States B and C are typically associated with one mode in the probability distribution function, because hazards are typically more rare, stronger, and systemic than for state A that is occurring regularly with a much smaller recurrence time. State $\mathrm{C}$ is typically associated with a critical dynamics that is characterized by a power-law distribution of system stressors and outcomes.

Such considerations suggest that the focus of complex system design and management — including "resilience management" — should be on reducing complexity to manageable levels required to achieve articulated goals and objectives (Jones, 2014). This can be achieved by improving traditional modeling methods, integrating these methods with real-time sensors, finding optimal design rules by investigating analogous systems, and mining relevant information from data. From this vantage point, the prescriptive import of the prevailing resilience paradigms require an important conceptual coda. To be sure, risk managers should seek to identify critical transitions, critical states, tipping points, and the like, wherever and whenever possible; still, the fact remains that much of this learning will occur in the wake of accidents and catastrophes. Rarely will "zero-event, zero-consequence" or even "early integration" approaches to resilient design be prudent or even feasible; in truth, in some instances, such approaches may even be counter-productive (Park et al., 2013).

As with resilience, risk can be independent of any hazard because risk is a function related to a definable set of relevant system outcomes (such as a structural failure, species extinction, or disease outbreak), which can occur without any external factor occurring or a clear failure of specific intrinsic factors. These risks, or decays in systems' performance, are related to systems' self-organization. However, systems' self-organization alone is not able to entirely alleviate our persistent inability to predict completely the whole spectrum of systems' outcomes. Hence, from this consideration it stems the need to include the "environmental noise" in mathematical models, which allow us to have a better representation of the combined self-organization-environment dynamics on complex systems. Therefore, risk and resilience are both evaluative functions of a desired performance (e.g., a "systemic ecosystem service"); both functions can be seen as the first derivative of the system's performance function, and their negative or positive sign determines their connotation of being risk or resilience. The same models can be used to evaluate risks as well as resilience about criteria that can have a positive or negative connotation, depending on how these criteria are viewed and managed. The dynamics of these criteria (e.g., resources to manage) are dependent on both intrinsic and extrinsic dynamics, and can be included into decision analytic frameworks and models 


\section{Conclusion}

Our discussion above has sought to offer a constructive commentary concerning important aspects of our evolving conceptions of, and approaches to, resilience. In so doing, we have availed ourselves of a number of disparate views of resilience, each of which provides a purview and lens through which to construe the syntax and semantics of different paradigmatic conceptions of resilience. We now offer a closing commentary that looks to provide a tentative outline of possible future research directions that takes the pluralistic conception of resilience set forth here as its point of departure.

To this end, we begin by noting that if resilience is a desired or sought after property of systems (i.e., of societies, cities, communities, etc), then, all things being equal, it seems reasonable to suppose that "stakeholders" - broadly construable to include all forms of life on this planet - will prefer to enjoy the benefits that resilience brings/provides sooner rather than later. Just how such benefits are to be quantified and evaluated is a problem that poses vexing challenges for system planners and risk managers. A partial list of such challenges will include the complex preference structures that are endemic to these systems, as well as the multifaceted nature of the potential benefits (arriving or realized over both short- and longer-term time horizons). To be sure, it is far too easy to suppose that the challenges that resilience poses for affected stakeholders are somehow surmountable by appealing to one paradigm, or by subscribing to a "one-size-fits-all" mentality as to how such problems might be thought through and addressed in practical terms. Our remarks here perhaps go some distance towards making the case that there are, in fact, a plurality of "paradigms" that are capable of informing our understanding of how such matters might best be framed and approached.

As we have observed in the numerous examples cited in our presentation above, each problem domain - be it infrastructure, public health, technology, and a host of others presents its own unique set of challenges and objectives. In this light, discussions about resilience "paradigms" are best grounded in carefully posed questions that are capable of forming the basis of research agendas that confront the problems and limitations of prevailing theories and methodologies. To illustrate, we close our discussion by suggesting four lines of research that hold the promise of expanding upon the pluralistic conception of resilience that we have outlined here:

- Computationally efficient models for better characterizing the stochastic structural and 
behavioral modalities of resilience in complex systems (e.g., identifying structural and functional scale-invariant factors responsible for emerging stable patterns, and factors that maintain or increase resilience, avoiding undesired critical transitions);

- Sensing and monitoring technologies, with emphasis on characterizing uncertainty, ignorance, and surprise (e.g., development of models capable of exploring how quick-shocks and pre-cursor events can lead to transitions of interest, given their relevance to low probability/high consequence outcomes);

- Improved methods for identifying and visualizing system drivers, especially in systems with complex dependencies and interactions;

- Analytic frameworks that combine theories of resilience with theories of intentionality and collective action.

Many of the research topics outlined above have a broadly construable participatory element, with the overarching goal of achieving a type of resilience that is arrived at by minimizing the frequency and magnitude of undesired system effects, via instruments oriented towards anticipation, sensing/monitoring, learning, and adaptation. Other research trajectories are, of course, possible, and entirely consistent with the spirit that underlies the pluralistic conception of resilience that we have sketched here. Ultimately, we must strive to confront the essential tension that arises out of our need to view resilience, on the one hand, as an intrinsic quality of all life forms on Earth and, on the other, as one of a number of viable instrumental means to a plurality of possible trajectories and desired outcomes for humankind. 


\section{${ }_{759}$ Acknowledgments}

760 M.C. gratefully acknowledges the support provided by funding from the Gi-CoRE Global

761 Station for Big Data and Cybersecurity (https://gi-core.oia.hokudai.ac.jp/gsb/) at

762 Hokkaido University (Japan), and the Microsoft AI for Earth Program grant "Bio-Hydro-

763 Geo Dynamics: Mapping Systemic Earth Risk" https://www.microsoft.com/en-us/ai/

764 ai-for-earth-grantsg. The authors thank two anonymous reviewers of this journal for

765 helpful comments and suggestions on an earlier draft of this paper. The views and opinions

766 expressed herein do not necessarily state or reflect those of the United States Government,

${ }_{767}$ the Japan Government or any agency thereof. 


\section{References}

Sean C. Anderson, Andrew B. Cooper, and Nicholas K. Dulvy. Ecological prophets: quantifying metapopulation portfolio effects. Methods in Ecology and Evolution, 4(10):971981, 2013. doi: 10.1111/2041-210X.12093. URL https://besjournals .onlinelibrary. wiley.com/doi/abs/10.1111/2041-210X.12093.

Per Bak. How nature works: the science of self-organized criticality. Springer Science \& Business Media, 2013.

Jayanth R Banavar, Amos Maritan, and Andrea Rinaldo. Size and form in efficient transportation networks. Nature, 399(6732):130, 1999.

Jayanth R Banavar, Todd J Cooke, Andrea Rinaldo, and Amos Maritan. Form, function, and evolution of living organisms. Proceedings of the National Academy of Sciences, 111 (9):3332-3337, 2014.

Y. Bar-Yam. From big data to important information. Complexity, 21:73-98, 2016. doi: 10.1002/cplx.21785.

Nicholas Beale, David G Rand, Heather Battey, Karen Croxson, Robert M May, and Martin A Nowak. Individual versus systemic risk and the regulator's dilemma. Proceedings of the National Academy of Sciences, 108(31):12647-12652, 2011.

Ulrich Beck. World at risk. Polity, 2009.

E Bertuzzo, Lorenzo Mari, L Righetto, Marino Gatto, Renato Casagrandi, M Blokesch, I Rodriguez-Iturbe, and A Rinaldo. Prediction of the spatial evolution and effects of control measures for the unfolding haiti cholera outbreak. Geophysical Research Letters, 38(6), 2011.

William Bialek, Ilya Nemenman, and Naftali Tishby. Predictability, complexity, and learning. Neural Comput., 13(11):2409-2463, 2001. doi: 10.1162/089976601753195969.

Javier Borge-Holthoefer, Nicola Perra, Bruno Gonçalves, Sandra González-Bailón, Alex Arenas, Yamir Moreno, and Alessandro Vespignani. The dynamics of information-driven coordination phenomena: A transfer entropy analysis. Science Advances, 2(4), 2016. doi: 10. 1126/sciadv.1501158. URL http://advances.sciencemag.org/content/2/4/e1501158 Stephen Carpenter, Kenneth Arrow, Scott Barrett, Reinette Biggs, William Brock, AnneSophie Crépin, Gustav Engström, Carl Folke, Terry Hughes, Nils Kautsky, et al. General resilience to cope with extreme events. Sustainability, 4(12):3248-3259, 2012. 
M Convertino, A Reddy, Y Liu, and C Munoz-Zanzi. Neutral early warning model, criticality, and eco-epidemiological scaling for infectious diseases. Science Advances, 2019. doi: inreview.

Matteo Convertino and Craig Hedberg. Intelli-food: cyberinfrastructure for real-time outbreak source detection and rapid response. In International Conference on Smart Health, pages 181-196. Springer, 2014.

Matteo Convertino and Yang Liu. Portfolio decision technology for designing optimal syndemic management strategies. In Complex Systems Design \& Management Asia, pages 223-234. Springer, 2016.

Matteo Convertino, Christy M Foran, Jeffrey M Keisler, Lynn Scarlett, Andy LoSchiavo, Gregory A Kiker, and Igor Linkov. Enhanced adaptive management: integrating decision analysis, scenario analysis and environmental modeling for the everglades. Scientific reports, 3:2922, 2013.

Matteo Convertino, Yang Liu, and Haejin Hwang. Optimal surveillance network design: a value of information model. Complex Adaptive Systems Modeling, 2(1):6, 2014. ISSN 2194-3206. doi: 10.1186/s40294-014-0006-8.

Matteo Convertino, Timothy R Church, Geary W Olsen, Yang Liu, Eddie Doyle, Clifford R Elcombe, Anna L Barnett, Leslie M Samuel, Iain R MacPherson, and Thomas R J Evans. Stochastic pharmacokinetic-pharmacodynamic modeling for assessing the systemic health risk of perfluorooctanoate (pfoa). Toxicological Sciences, 2018. doi: 10.1093/toxsci/kfy035.

J. P. Crutchfield. Between order and chaos. Nature Physics, 8:17-24, 2012. doi: 10.1038/ nphys2190.

Susan L Cutter, Joseph A Ahearn, Bernard Amadei, Patrick Crawford, Elizabeth A Eide, Gerald E Galloway, Michael F Goodchild, Howard C Kunreuther, Meredith Li-Vollmer, Monica Schoch-Spana, et al. Disaster resilience: A national imperative. Environment: Science and Policy for Sustainable Development, 55(2):25-29, 2013.

Antonio Damasio and Gil B Carvalho. The nature of feelings: evolutionary and neurobiological origins. Nature reviews neuroscience, 14(2):143, 2013.

Olivier L De Weck, Daniel Roos, and Christopher L Magee. Engineering systems: Meeting human needs in a complex technological world. Mit Press, 2011.

Jared (Jared Mason) Diamond. Collapse: how societies choose to fail or succeed. Viking Penguin, 2005. 
Rainer Feistel and Werner Ebeling. Entropy and the self-organization of information and value. Entropy, 18(5), 2016. doi: 10.3390/e18050193. URL http://www.mdpi.com/ $1099-4300 / 18 / 5 / 193$.

Carl Folke. Resilience: The emergence of a perspective for social-ecological systems analyses. Global environmental change, 16(3):253-267, 2006.

Alexander A Ganin, Emanuele Massaro, Alexander Gutfraind, Nicolas Steen, Jeffrey M Keisler, Alexander Kott, Rami Mangoubi, and Igor Linkov. Operational resilience: concepts, design and analysis. Scientific reports, 6:19540, 2016.

Nikunj Goel, Vishwesha Guttal, Simon Levin, and Carla Staver. Dispersal increases the resilience of tropical savanna and forest distributions. bioRxiv, page 476184, 2018.

Andrew G Haldane and Robert M May. Systemic risk in banking ecosystems. Nature, 469 (7330):351, 2011.

D. Helbing, D. Brockmann, T. Chadefaux, K. Donnay, U. Blanke, O. Woolley-Meza, M. Moussaid, A. Johansson, J. Krause, S. Schutte, and M. Perc. Saving Human Lives: What Complexity Science and Information Systems can Contribute. Journal of Statistical Physics, 158:735-781, 2015. doi: 10.1007/s10955-014-1024-9.

Dirk Helbing. Globally networked risks and how to respond. Nature, 497(7447):51-59, 口 2013. ISSN 0028-0836. doi: 10.1038/nature12047. URL http://dx.doi.org/10.1038/ nature12047.

Jorge Hidalgo, Jacopo Grilli, Samir Suweis, Amos Maritan, and Miguel A Munoz. Cooperation, competition and the emergence of criticality in communities of adaptive systems. Journal of Statistical Mechanics: Theory and Experiment, 2016(3), 2016. URL http://stacks.iop.org/1742-5468/2016/i=3/a=033203.

Crawford S Holling and Lance H Gunderson. Resilience and adaptive cycles. In: Panarchy:

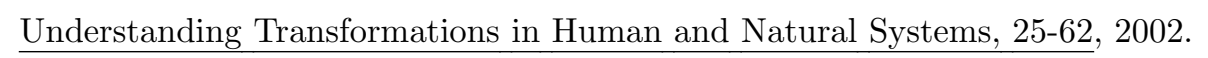

Chirag J Patel and Arjun K Manrai. Development of exposome correlation globes to map out environment-wide associations. Pacific Symposium on Biocomputing. Pacific Symposium on Biocomputing, 20:231-42, 2015. doi: 10.1142/9789814644730_0023.

Raúl Jiménez, Haydee Lugo, José A Cuesta, and Angel Sánchez. Emergence and resilience of cooperation in the spatial prisoner's dilemma via a reward mechanism. Journal of theoretical biology, 250(3):475-483, 2008. 
Peter H Jones. Systemic design principles for complex social systems. In Social systems and design, pages 91-128. Springer, 2014.

Stuart A Kauffman. The origins of order: Self-organization and selection in evolution. OUP USA, 1993.

Sonia Kéfi, Vasilis Dakos, Marten Scheffer, Egbert H Van Nes, and Max Rietkerk. Early warning signals also precede non-catastrophic transitions. Oikos, 122(5):641-648, 2013.

Sonia Kefi, Vishwesha Guttal, William A Brock, Stephen R Carpenter, Aaron M Ellison, Valerie N Livina, David A Seekell, Marten Scheffer, Egbert H van Nes, and Vasilis Dakos. Early warning signals of ecological transitions: methods for spatial patterns. PloS one, 9 (3):e92097, 2014.

Loren Koçillari, Piero Fariselli, Antonio Trovato, Flavio Seno, and Amos Maritan. Signature of pareto optimization in the escherichia coli proteome. Scientific reports, 8(1):9141, 2018.

J. Stephen Lansing, Stefan Thurner, Ning Ning Chung, Aurélie Coudurier-Curveur, Çăgil Karakaş, Kurt A. Fesenmyer, and Lock Yue Chew. Adaptive self-organization of bali's ancient rice terraces. Proceedings of the National Academy of Sciences, 114(25):65046509, 2017. ISSN 0027-8424. doi: 10.1073/pnas.1605369114. URL https://www.pnas. org/content/114/25/6504.

SA Levin. Fragile dominion: complexity and the commons. perseus. Reading, MA, 1999.

Simon A Levin and Jane Lubchenco. Resilience, robustness, and marine ecosystem-based management. Bioscience, 58(1):27-32, 2008.

J Li and M Convertino. Optimal microbiome networks: Macroecology and criticality. Entropy, 2019. doi: inpress.

I. Linkov and B.D. Trump. The Science and Practice of Resilience. Risk, Systems and Decisions. Springer International Publishing, 2019. ISBN 9783030045630. URL https: //books.google.com/books?id=nAZJvQEACAAJ.

I. Linkov, T. Bridges, F. Creutzig, J. Decker, C. Fox-Lent, W. Kröger, J. H. Lambert, A. Levermann, B. Montreuil, J. Nathwani, R. Nyer, O. Renn, B. Scharte, A. Scheffler, M. Schreurs, and T. Thiel-Clemen. Changing the resilience paradigm. Nature Climate Change, 4:407-409, 2014. doi: 10.1038/nclimate2227.

Igor Linkov, Benjamin D Trump, and Jeffrey Keisler. Risk and resilience must be independently managed. Nature, 555(7694), 2018. 
Matteo Marsili, Iacopo Mastromatteo, and Yasser Roudi. On sampling and modeling complex systems. Journal of Statistical Mechanics: Theory and Experiment, 2013(09), 2013.

Emanuele Massaro, Alexander Ganin, Nicola Perra, Igor Linkov, and Alessandro Vespignani. Resilience management during large-scale epidemic outbreaks. Scientific reports, 8(1):1859, 2018.

Jeryang Park, Thomas P Seager, Palakurth Suresh Chandra Rao, Matteo Convertino, and Igor Linkov. Integrating risk and resilience approaches to catastrophe management in engineering systems. Risk Analysis, 33(3):356-367, 2013.

Stephen G. Perz, Rafael Munoz-Carpena, Gregory Kiker, and Robert D. Holt. Evaluating ecological resilience with global sensitivity and uncertainty analysis. Ecological Modelling, 263:174 - 186, 2013. ISSN 0304-3800. doi: https://doi.org/10.1016/j.ecolmodel.2013.04. 024.

Mikhail Prokopenko, Fabio Boschetti, and Alex J. Ryan. An informationtheoretic primer on complexity, self-organization, and emergence. Complexity, 15(1):11-28, 2008. doi: $10.1002 /$ cplx.20249.

R. Quax, O. Har-Shemesh, S. Thurner, and P. M. A. Sloot. Stripping syntax from complexity: An information-theoretical perspective on complex systems. ArXiv e-prints, 2016.

Juan C Rocha, Garry Peterson, Örjan Bodin, and Simon Levin. Cascading regime shifts within and across scales. Science, 362(6421):1379-1383, 2018.

Johan Rockström, Will Steffen, Kevin Noone, Åsa Persson, F Stuart Chapin III, Eric F Lambin, Timothy M Lenton, Marten Scheffer, Carl Folke, Hans Joachim Schellnhuber, et al. A safe operating space for humanity. nature, 461(7263):472, 2009.

Casey Sarapas, Guiqing Cai, Linda M Bierer, Julia A Golier, Sandro Galea, Marcus Ising, Theo Rein, James Schmeidler, Bertram Müller-Myhsok, Manfred Uhr, et al. Genetic markers for ptsd risk and resilience among survivors of the world trade center attacks. Disease markers, 30(2, 3):101-110, 2011.

Marten Scheffer, Steve Carpenter, Jonathan A Foley, Carl Folke, and Brian Walker. Catastrophic shifts in ecosystems. Nature, 413(6856):591, 2001.

Marten Scheffer, Stephen R Carpenter, Timothy M Lenton, Jordi Bascompte, William Brock, Vasilis Dakos, Johan Van de Koppel, Ingrid A Van de Leemput, Simon A Levin, Egbert H Van Nes, et al. Anticipating critical transitions. science, 338(6105):344-348, 2012. 
Marten Scheffer, J Elizabeth Bolhuis, Denny Borsboom, Timothy G Buchman, Sanne MW Gijzel, Dave Goulson, Jan E Kammenga, Bas Kemp, Ingrid A van de Leemput, Simon Levin, et al. Quantifying resilience of humans and other animals. Proceedings of the National Academy of Sciences, 115(47):11883-11890, 2018.

Luís F Seoane and Ricard Solé. Systems poised to criticality through pareto selective forces. arXiv preprint arXiv:1510.08697, 2015.

Joseph L. Servadio and Matteo Convertino. Optimal information networks: Application for data-driven integrated health in populations. Science Advances, 4(2), 2018. doi: 10.1126/ sciadv.1701088.

Cosma Rohilla Shalizi and James P. Crutchfield. Computational mechanics: Pattern and prediction, structure and simplicity. Journal of Statistical Physics, 104(3):817-879, 2001. ISSN 1572-9613. doi: 10.1023/A:1010388907793. URL https://doi.org/10.1023/A: 1010388907793 .

Yossi Sheffi et al. The resilient enterprise: overcoming vulnerability for competitive advantage. MIT Press Books, 1, 2005.

Avichai Tendler, Avraham Mayo, and Uri Alon. Evolutionary tradeoffs, pareto optimality and the morphology of ammonite shells. BMC systems biology, 9(1):12, 2015.

Kathleen Tierney. The social roots of risk: Producing disasters, promoting resilience. Stanford University Press, 2014.

Max Troell, Rosamond L Naylor, Marc Metian, Malcolm Beveridge, Peter H Tyedmers, Carl Folke, Kenneth J Arrow, Scott Barrett, Anne-Sophie Crépin, Paul R Ehrlich, et al. Does aquaculture add resilience to the global food system? Proceedings of the National Academy of Sciences, 111(37):13257-13263, 2014.

L. James Valverde and Matteo Convertino. Insurer resilience in an era of climate change and extreme weather: An econometric analysis. Climate, 7(4), 2019. ISSN 2225-1154. doi: 10.3390/cli7040055. URL http://www.mdpi.com/2225-1154/7/4/55.

Maarten van Strien, Sibyl Huber, John Anderies, and Adrienne Grêt-Regamey. Resilience in social-ecological systems: identifying stable and unstable equilibria with agent-based models. Ecology and Society, 24(2), 2019.

Victor Del Rio Vilas, M Kocaman, Howard Burkom, Richard Hopkins, John Berezowski, Ian Painter, Julia Gunn, G Montibeller, M Convertino, LC Streichert, et al. A value-driven 

framework for the evaluation of biosurveillance systems. Online journal of public health informatics, 9(1), 2017.

${ }_{957}$ Geoffrey B West, James H Brown, and Brian J Enquist. A general model for the origin of allometric scaling laws in biology. Science, 276(5309):122-126, 1997.

${ }_{959}$ Young Ae You, Mohammad Ali, Suman Kanungo, Binod Sah, Byomkesh Manna, Mahesh 960 Puri, G Balakrish Nair, Sujit Kumar Bhattacharya, Matteo Convertino, Jacqueline L Deen, 961 et al. Risk map of cholera infection for vaccine deployment: the eastern kolkata case. PloS $962 \quad$ one, 8(8):e71173, 2013. 


\section{Figure Captions}

\section{Figure 1. Holistic Conception of Collective Phenomena Leading to Scale-Free}

Resilient Networks. Individuals self-organize around collective decisions that result in emerging patterns. These patterns, such as patterns of human mobility (the middle plot represents average scale-free mobility fluxes in NYC from and to Manhattan using the shortest path tree from any location to another), are the by-product of (bottom-line) local interactions among individuals constrained on a mobility infrastructure (i.e., multiple sets of transportation networks), typically designed by structured, top-down decisions. Top-down decisions are planned via decision-analytic models (bottom of the figure, for instance) that can integrate stakeholder needs and preferences, and network features. The spontaneous criticality of living systems enhanced by critical decision-making can sustain optimal complex system performance and resilience trajectories. As is the case for natural networks, the local search for local minimum energy expenditure under global constraints and objective(s), leads to optimal scale-free networks.

Figure 2. General Conception of Pluralistic Resilience for Complex Systems. (A) Complexity, sensitivity, and uncertainty morphospace for complex systems. These features of complex systems can be related to systems' cognition, spatio-temporal scale, and entropy that defines the information of any system (e.g., data) or models (as "information machines") representing the real systems. (B) Different conceptions of resilience proposed in the literature: single event system's response curve and performance-driver system's landscape in the left and middle plots. The right plot shows our conception of resilience, where the pdf of system's state is evaluated in different time periods; the transition between pdfs toward desired system's performance reflects system's resilience. The same temporal consideration, i.e., how the system responds to stressors, can be made for the other two resilience views, but fails to consider the long-term trajectory, the whole spectrum of stressors, intrinsic system's drivers including stakeholders' preferences, and surprises.

Figure 3. Long-term Trajectories of System's Performance. The change from one system (average) performance state to another typically involves a long-time span, which is different from the short-term response to stressors that may show a high degree of nonlinearity (e.g., small stressors can cause large effects). The change from one single stressor state to another is typically associated with an alteration of system function (or, more holistically, performance), observable in the increased variance of system components (depicted as change of node color, from white to red), before the change occurs. For major transitions, 
this change is also observable in the high variance of system structure (e.g., connectivity among nodes). A "resilient" stable state generally corresponds to a low variance critical dynamics (e.g., network with "white nodes"), but mutable in case of necessity. The black trajectory shows a resilient system that increases system performance over time, versus a resistant system that recovers from point stressors, but does not increase the performance over time (green trajectory), and a anti-resilience system (orange trajectory) that does decrease system's performance over time. The pdf of system's performance (Fig. 2 ) for the black trajectory has the highest, most positive, and least uncertain performance.

Figure 4. Network-Based Dynamical System Classification. Undirected or weakly directed networks (left), where one node is dependent on many independent nodes, is typical for linear systems. A highly direct network (right), where any node is dependent on many interdependent nodes, is typical for non-linear systems. The former can be the case of a river network, while the latter can be a biological network such as the microbiome. A risk and resilience approach is the best approach for the linear and the non-linear system. Node 1 can be thought of as the performance function to predict.

Figure 5. Characterization of System Dynamics via Global Sensitivity and Uncertainty Analyses. Global Sensitivity and Uncertainty Analyses (GSUA) plot (left) characterizes complex systems according to their dynamics with respect to a predicted performance (e.g., node 1 in the networks depicted in Fig. 4). Red nodes (i.e., model/system factors) represent a system with critical dynamics, while blue and green nodes represent a system with a linear (deterministic) and non-linear (chaotic) dynamic, respectively. Critical systems are systems characterized by scale-free dynamics that ensure high resilience. pdfs and time series on the right of the GSUA plot show the typical dynamics for different systems.

Figure 6. Information Selection of Resilient Systems. Scaling analysis of total information as a function of the information threshold. When a system transitions from one phase to another, it loses or gains symmetry. In this context there is an ideal scale-invariant region for which the total information (i.e., the uncertainty) that a system can gain about a system's dynamics is the same. However, for low values of the information threshold (defining the sensitivity of the system) more redundancy of information exists. This can lead to a loss of information or to a higher risk of predicting risk without accuracy, due to the inclusion of irrelevant nodes. The maximum value of the information entropy has the highest utility for a decision-maker in terms of system's predictability. Complexity is related to network com- 
plexity determined by the diverse set of functional network properties. Lowest complexity and highest sensitivity are typically associated with each other, due to the lack of redundant nodes.

Figure 7. Epidemiological Epitome of Complex System Dynamics. Spatial network topology (of a functional nature, considering the interdependence of cases) is important in determining the dynamics of complex systems, and also in determining areas with high risk and in defining systems' resilience. In epidemiology, high risk areas are associated with persistent, large outbreaks. The persistent "bouncing-back" dynamics of these critical areas has nothing to do with the ability of the population to respond to outbreaks, but rather, is related to the intrinsic dynamics of the disease. Criticality is, in such cases, an undesired property. Resilient communities are more likely those that sustain low levels of the disease, such as the community highlighted in green. These communities tend to be more isolated, but their undesired critical performance is avoided by the isolation versus at-risk communities that are hubs of scale-free networks (e.g., the purple community). The blue network defined on the map is the optimal transmission network, which is the directed scale-free network (toward the capital community Colombo, depicted as the black node on the map), assessed by using the maximum transfer entropy algorithm of Servadio and Convertino $(2018)$ on the epidemiological patterns of Leptospirosis in Sri Lanka.

Figure 8. Potential System Landscape. The system landscape represents all potential states of the system (and trajectories from one state to another) that are identified by system's performance pdf, as a function of a multicriteria driver function (stressors, vulnerability, and exposure functions, derivable using traditional risk analysis methods). Stable resilient states are characterized by small total energy dissipation, minimum entropy, and low probability of system's transitions toward unstable states. These resilient states have also high free energy, which allows them to change state in case of need. The whole system landscape can be mapped via uncertainty methods, such as information-theoretic GSUA that identifies all potential system states (e.g., A, B, and C among all others) and the corresponding pdfs defining the likelihood of events to occur at different scales (e.g., for the whole system and for subcomponents). 


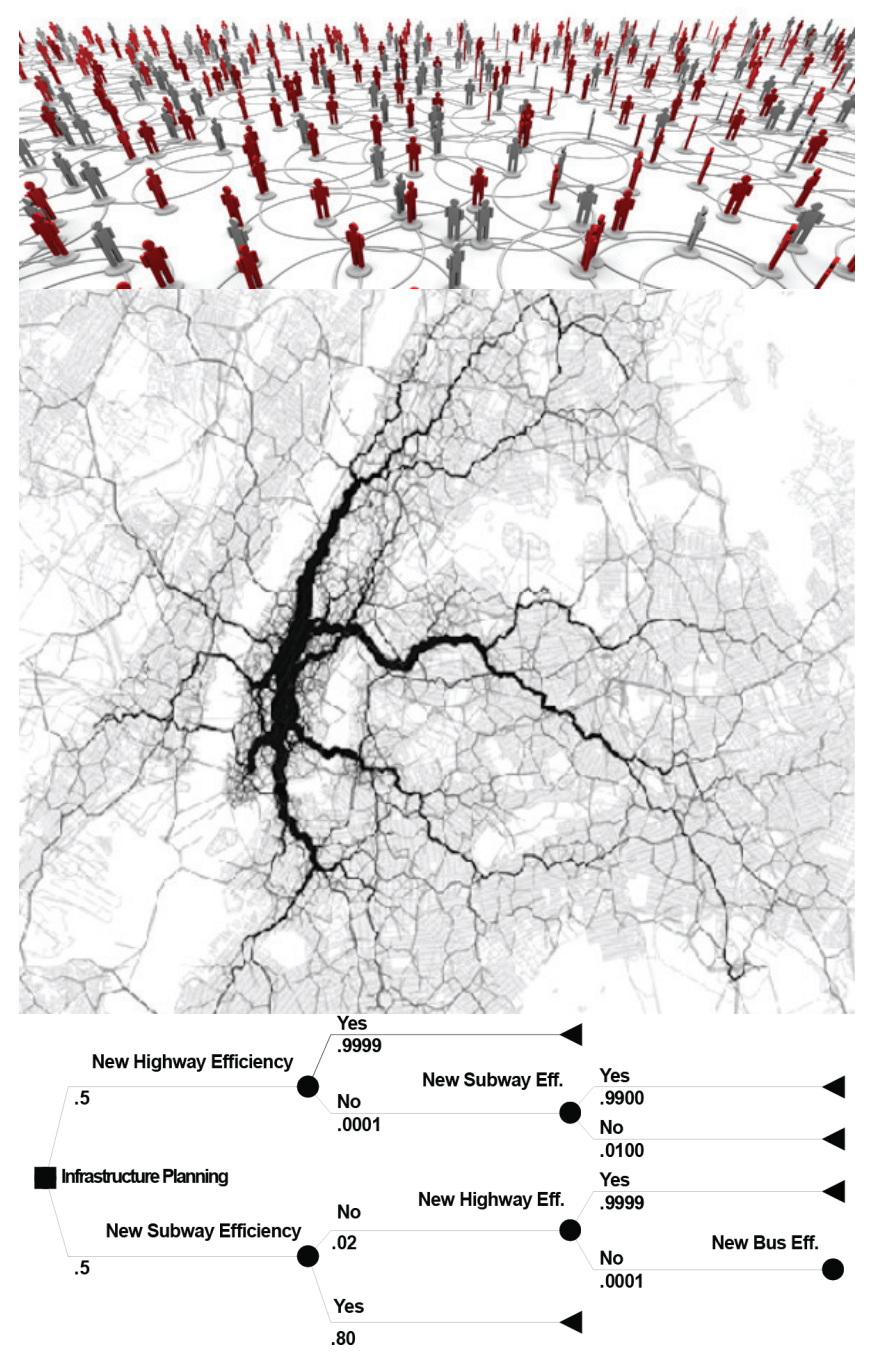

Figure 1: 

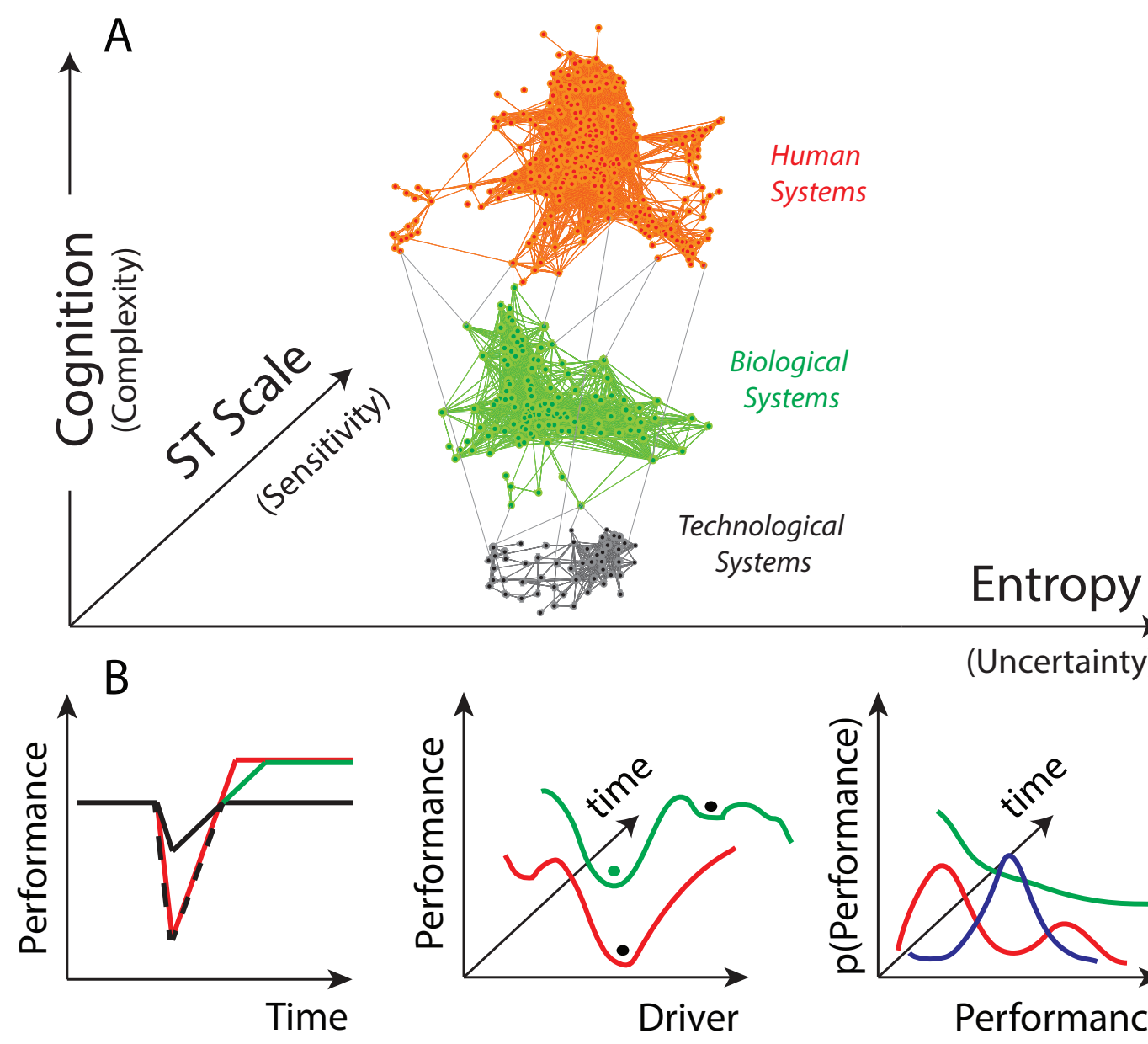

(Uncertainty)
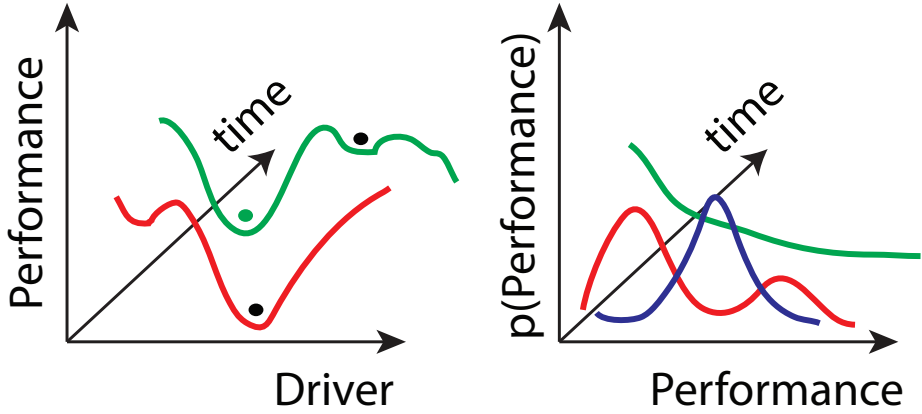

Figure 2: 


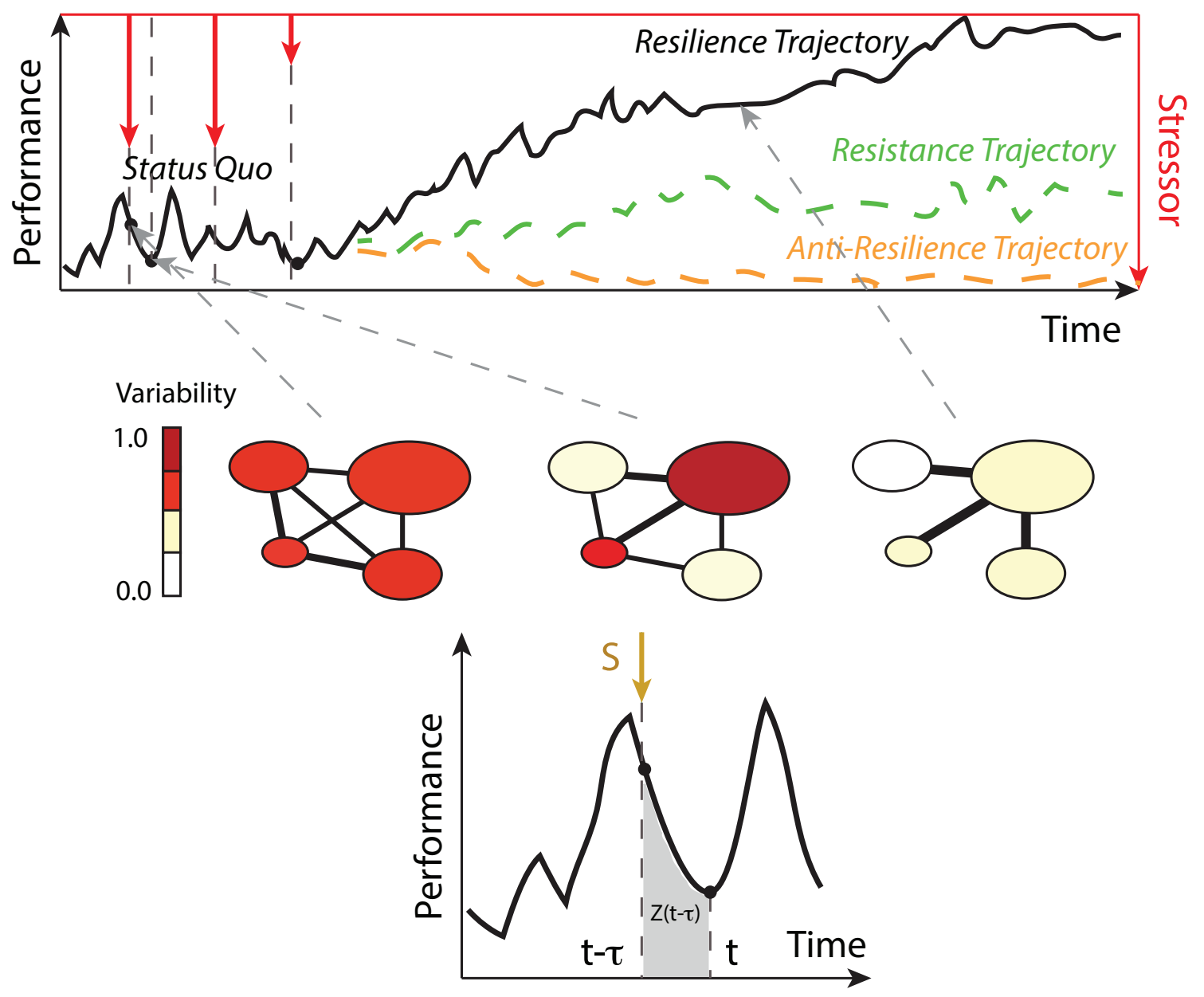

Figure 3: 
Risk Approach (Linear System)

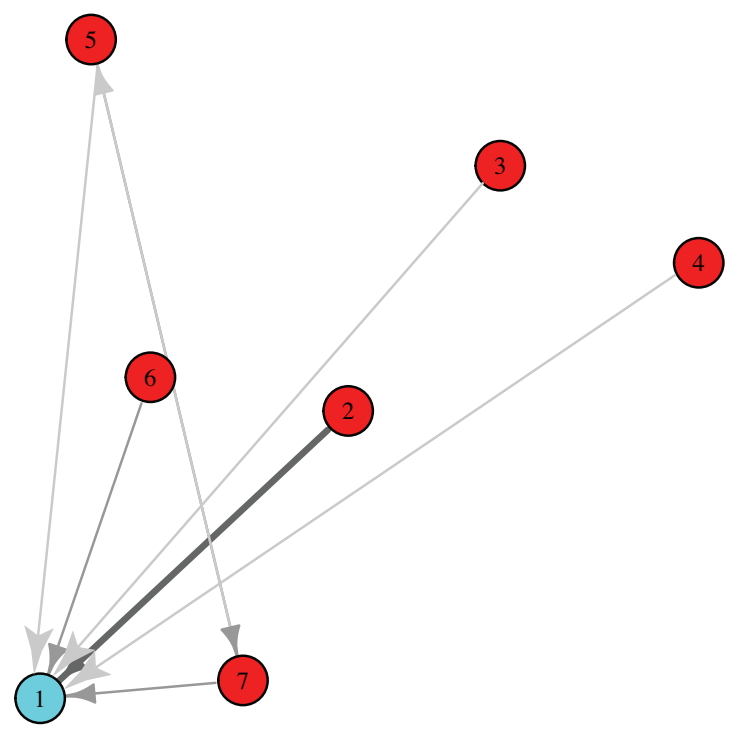

Resilience Approach (Non-Linear System)

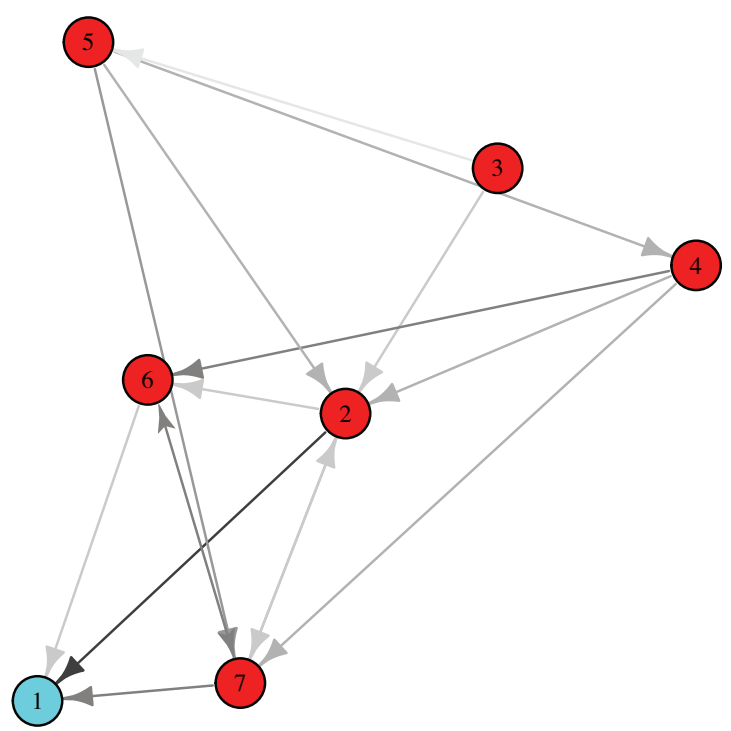

Figure 4: 

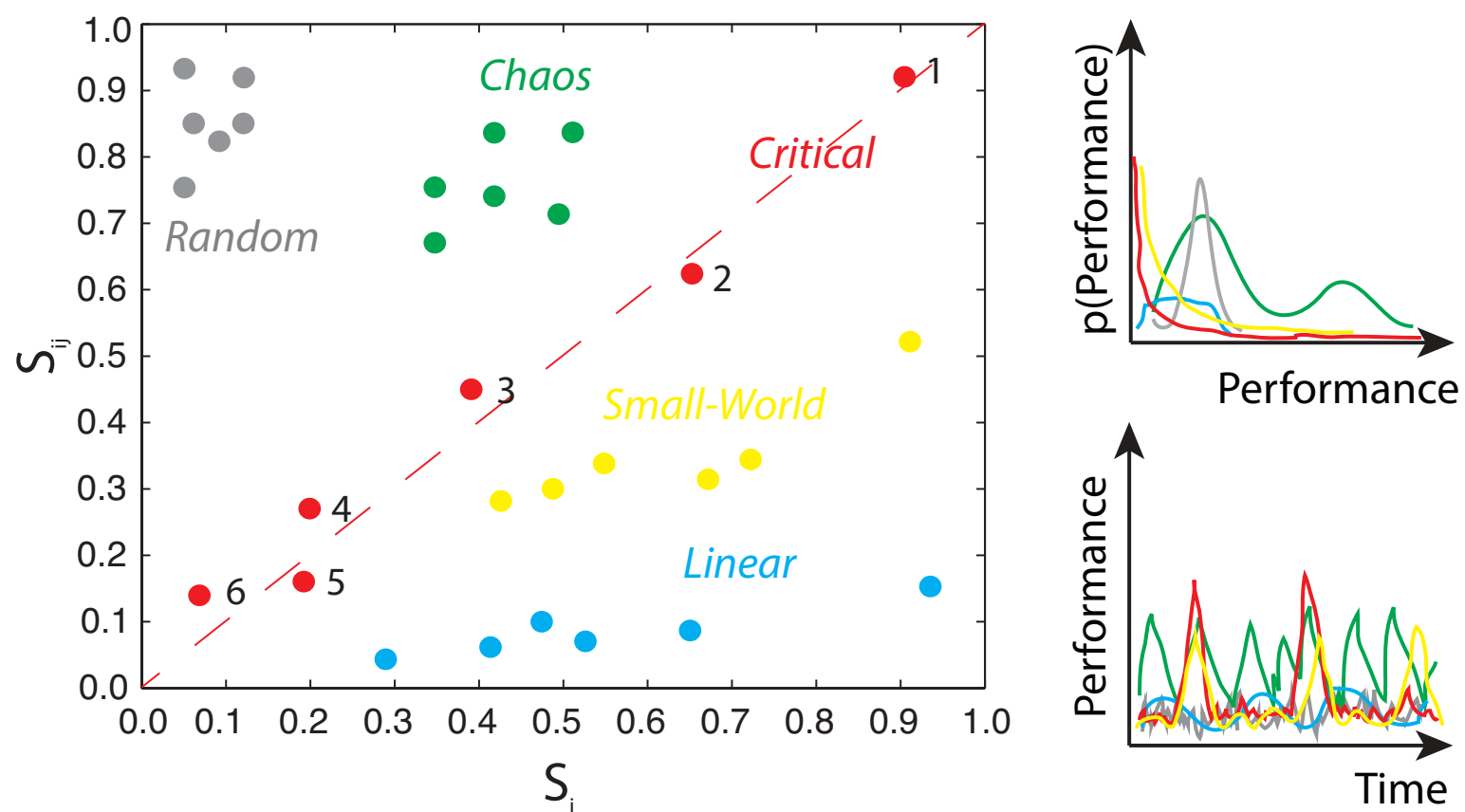

Figure 5: 


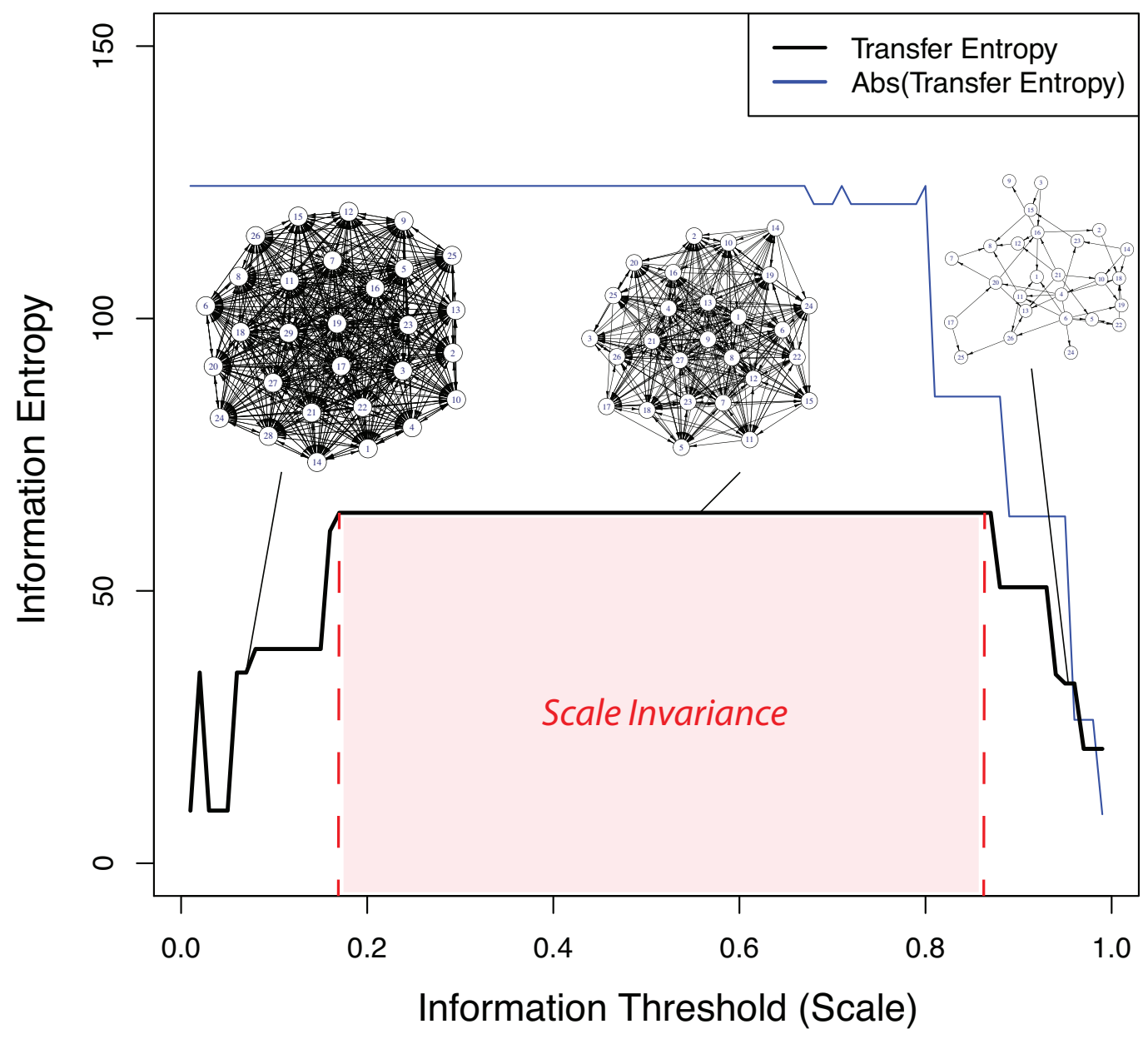

Figure 6: 

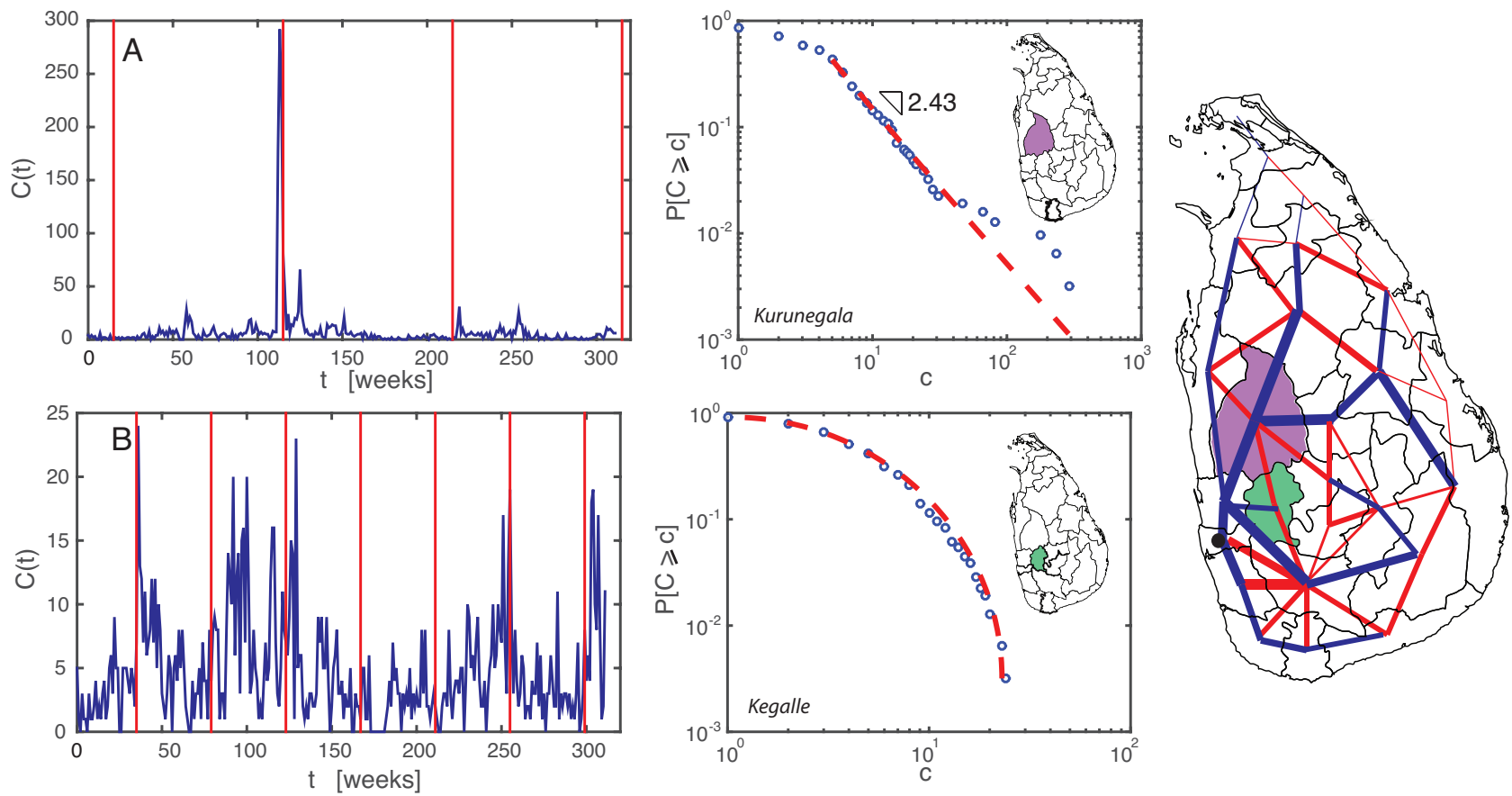

Figure 7: 

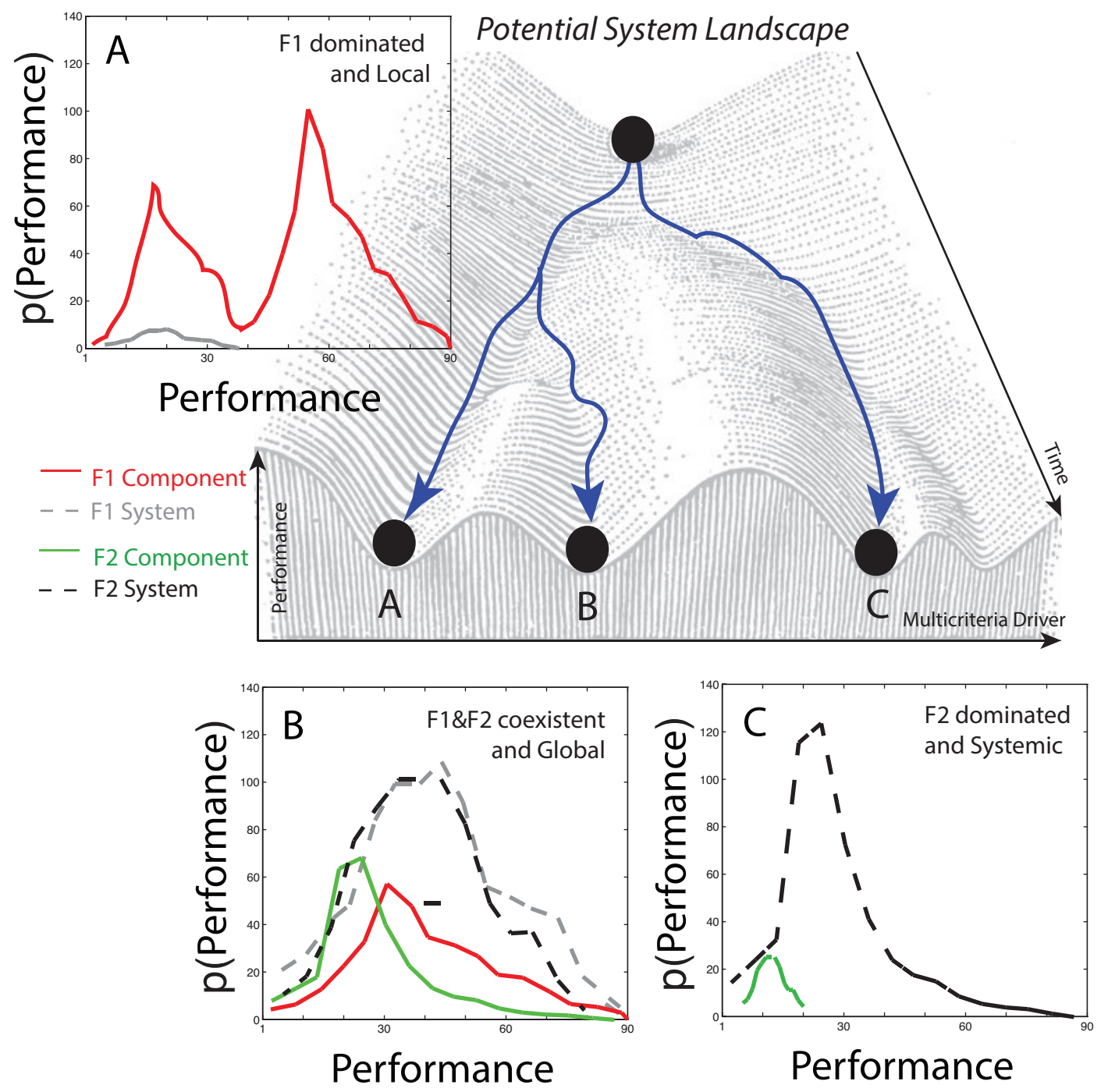

Figure 8: 